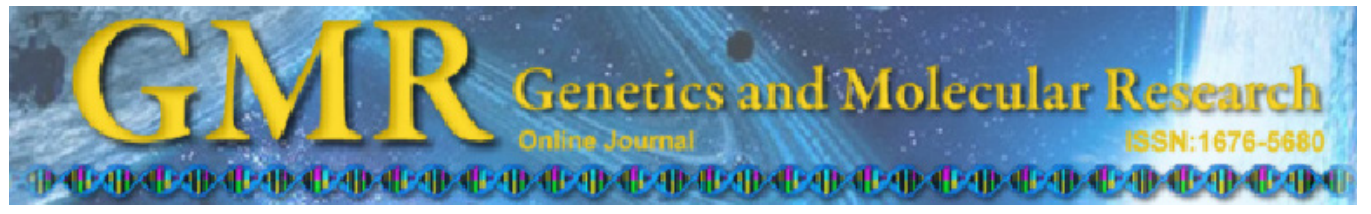

\title{
Screening and functional microarray analysis of differentially expressed genes related to osteoporosis
}

\author{
Y. Chen and R.G. Xia \\ Shanghai, China \\ Corresponding author: R.G. Xia \\ E-mail: xiaronggangxrg@hotmail.com \\ Genet. Mol. Res. 13 (2): 3228-3236 (2014) \\ Received December 10, 2012 \\ Accepted June 14, 2013 \\ Published April 25, 2014 \\ DOI http://dx.doi.org/10.4238/2014.April.25.8
}

Department of Orthopedics, Sixth People's Hospital of Shanghai,

\begin{abstract}
We searched for key genes that could accurately predict bone mineral density. The gene expression profile GSE7429 was downloaded from the Gene Expression Omnibus database, which includes 20 samples, 10 with high and 10 with low bone mineral density. The differentially expressed genes (DEGs) were identified with packages in $\mathrm{R}$ language. Further, BLASTX was used to obtain COG function classifications of all the DEGs. The GOTM software was used to find DEGs enriched modules. The functions of genes in the modules was also predicted with the software GENECODIS. Three hundred and three genes were identified as DEGs by comparing high and low bone mineral density samples; the selected genes were mapped to 14 modules collected in PPID. Genes VDR, ESR1, and NRIP1, located in the same module, were significantly enriched in intracellular receptor-mediated signaling biological processes. We conclude that the genes VDR, ESR1, NRIP1 in B cells have a close relationship with bone mineral density. The expression patterns of these genes could be used to determine osteoprotegerin function and for early diagnosis and prevention of low bone mineral density.
\end{abstract}

Key words: Bone mineral density; B cell; Functional analysis; Differentially expressed genes; Enrichment analysis 


\section{INTRODUCTION}

Osteoporosis is the most common and serious skeletal disorder among the elderly and postmenopausal women, and it is characterized by reduced bone mineral density (BMD) and bone microarchitecture degeneration, leading to increased fracture risk (Peacock et al., 2002). The measurement of BMD has been considered for years to be the "gold standard" in osteoporosis diagnosis (Kiel et al., 2007).

BMD is known to be highly heritable, and an increasing number of genes have been identified to have independent effects on BMD in osteoporosis, including pre-B-cell leukemia homeobox 1 (PBX1) (Cheung et al., 2009), apolipoprotein E (APOE) (Singh et al., 2010), Jagged1 (JAG1) (Kung et al., 2010), TWIST1 gene (Hwang et al., 2010), TNFRSF11B (osteoprotegerin) (Vidal et al., 2011), and SOX6 (Yang et al., 2012). In addition, in osteoporotic women, femoral neck BMD shows associations with vitamin D receptor gene (VDR), and lumbar spine BMD with osteoprotegerin (OPG) and tumor necrosis factor superfamily member 11 (TNFSF11) (Mencej-Bedrač et al., 2009). The interaction between genetic variants in the CD40 and CD40L genes has been demonstrated to exert a role in reducing BMD and increases osteoporosis risk in women. Periostin (POSTN), an extracellular matrix protein secreted by osteoblasts and a regulator of osteoblast differentiation and bone formation, also affects susceptibility to low BMD and osteoporosis (Xiao et al., 2012). Importantly, a study by Duncan et al. (2011) suggests six new genetic associations in or around the genes CLCN7, GALNT3, IBSP, LTBP3, RSPO3, and SOX4. Loss-offunction gene mutation in GALNT3 causes high bone mass.

However, high throughput screening the differentially expressed genes (DEGs) between high and low bone mineral density samples and function analysis of them remain rare. In this study, we aimed to first screen DEGs between high and low bone mineral density samples by using a microarray dataset available from a public database. Furthermore, the underlying molecular mechanisms of these crucial genes were predicted by biological process enrichment analysis.

\section{MATERIAL AND METHODS}

\section{Affymetrix microarray data}

The GSE7429 dataset was download from the National Center for Biotechnology Information Gene Expression Omnibus data repository (http://www.ncbi. nlm.nih.gov/geo/). This dataset includes 20 samples from unrelated postmenopausal white women, 10 with high and 10 with low bone mineral density [GPL96 record: (HG-U133A) Affymetrix Human Genome U133A Array] (Xiao et al., 2008). This study was approved by the Institutional Review Board, and all the subjects signed informed consent documents before entering the project. All the study subjects were whites of European origin recruited from the vicinity of Creighton University in Omaha, NE, USA.

\section{Data preprocessing and analysis of DEGs}

The limma method (Xiao et al., 2008) was used to identify DEGs in blood B 
cells from different bone mineral density samples. The original expression datasets from all conditions were processed into expression estimates using packages in $\mathrm{R}$ language (Troyanskaya et al., 2001; Fujita et al., 2006). Significance of gene expression differences was tested by the classical $t$-test, and the $\mathrm{P}$ values were adjusted for multiple comparisons using Bayes' theorem (Benjamini and Hochberg, 1995). Only the genes showing $\mathrm{P}<0.01$ were selected as DEGs.

\section{Gene function analysis}

The sequence alignment of DEGs with the COGs (clusters of orthologous groups of proteins) database was carried out by BLASTX (E value $<1 \mathrm{e}-05$ ) (Wootton and Federhen, 1993). Functional annotation and COG functional classification of DEGs were identified from cellular component, molecular function, and biological process. COG functional classification could give intuitive and rational understanding about DEGs function.

\section{DEGs module enrichment analysis}

Under normal conditions, genes cannot carry out their function alone but only if they interact with other factors. In this study, module enrichment analysis of DEGs based on the hypergeometric distribution algorithm module was performed using gene ontology tree machine (GOTM) software (adj P < 0.05) (Zhang et al., 2005).

\section{Biological process enrichment analysis of DEGs}

GeneCodis is a web server application for functional analysis of gene lists that integrates different sources of information (GO, KEGGor or SwissProt) and finds modular patterns of interrelated annotations (Nogales-Cadenas et al., 2009). The GENECODIS software (adj $\mathrm{P}<0.05$ ) was used to analyze the differentially expressed biological process genes (Tabas-Madrid et al., 2012).

\section{RESULTS}

\section{Identifying DEGs}

After data preprocessing, gene expression profile data with higher normalization were used for DEGs analysis (Figure 1). We identified 303 DEGS that met the threshold $(\mathrm{P}<0.01)$.

\section{Functional classification of DEGs}

BLASTX was used to align sequence of 303 DEGs with the COGs database (E value $<1 \mathrm{e}-05)$. Functional classification was based on the degree of sequence alignment of DEGS in the Gene Ontology database (Figure 2). The function of DEGs included cellular component, molecular function and biological process. 


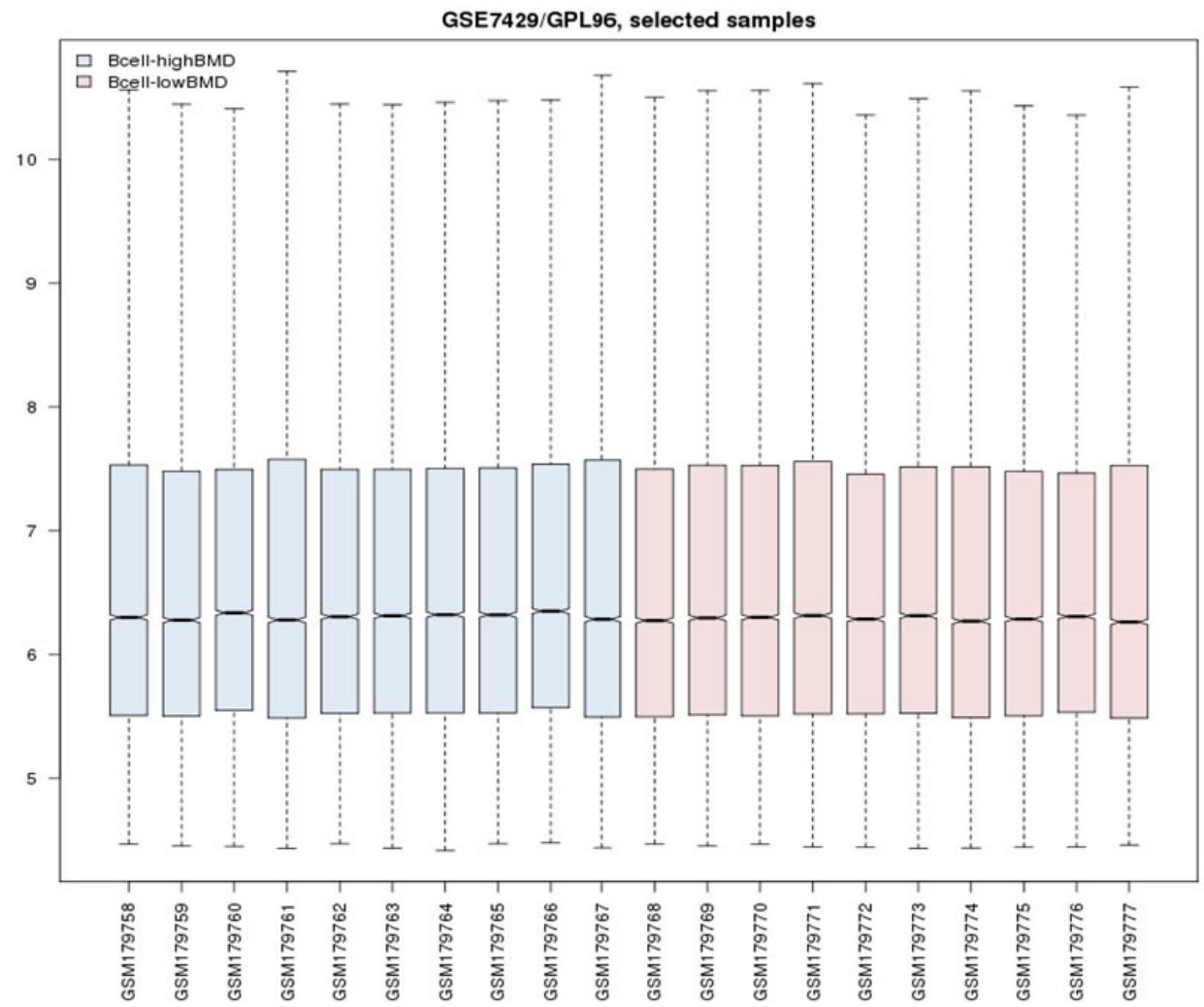

Figure 1. Microarray data normalization. Ten high bone mineral density (blue boxes) and 10 low bone mineral density (pink boxes) microarray data are normalized. The black line in every box is the median which decide the level of normalization of every data. Black line of median is in a straight line, which indicates a good median normalization.
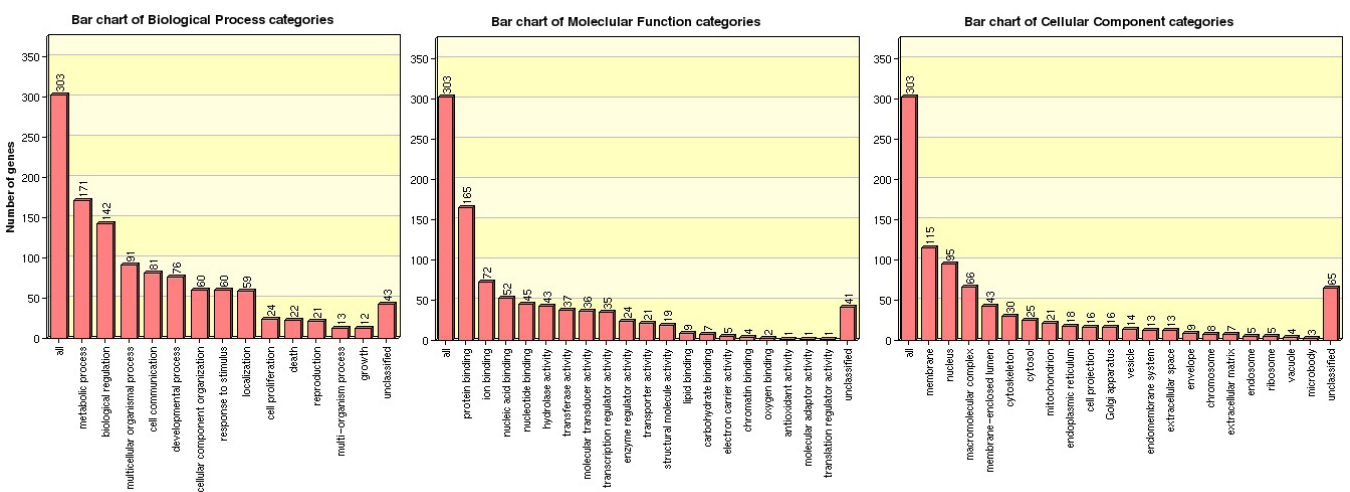

Figure 2. Classification of 303 differentially expressed genes. From left to right: biological process, molecular function, and cellular component. X-coordinate indicates different biological process, molecular function, cell constituent. Y-coordinate indicates the number of differentially expressed gene. 


\section{DEGs module enrichment analysis}

GOTM was used to perform module enrichment analysis of DEGs on the basis of the hypergeometric distribution algorithm (adj $\mathrm{P}<0.05)$. A total of 14 major functional enrichment modules were obtained (Table 1).

\section{Biological process analysis of DEGs}

We analyzed the biological process for DEGS in all 14 major functional enrichment modules. The results showed that these genes were mainly involved in 7 biological processes, including the intracellular receptor-mediated signaling pathway, interaction with symbiont, regulation of retinoic acid receptor signaling pathway, polysaccharide transport, retinoic acid receptor signaling pathway, positive regulation of retinoic acid receptor signaling pathway, and lipopolysaccharide transport (Table 2). Among them, intracellular receptor-mediated signaling pathway (GO: 0030522) was the most significant biological process, involving the VDR, ESR1 (estrogen receptor 1), and NRIP1 (nuclear receptor interacting protein 1) genes, which formed a module (DB_ID: 900). Further analysis indicated that the expression of VDR and ESR1 were dramatically decreased in blood B cells from low bone mineral density samples. In contrast, the expression of NRIP1 was increased in blood B cells from low bone mineral density samples (Figure 3).

\begin{tabular}{|c|c|c|c|}
\hline DB_ID & Raw P & Adjusted P & Gene \\
\hline 1039 & $2.91 \mathrm{E}-05$ & 0.0004 & GJC2, TLN2, ALB, TRY6, AGA \\
\hline 730 & $4.41 \mathrm{E}-05$ & 0.0004 & PPP2CA, ISYNA1, PPP2R4 \\
\hline 871 & 0.0011 & 0.0043 & ZNF410, MYO9A \\
\hline 731 & 0.0011 & 0.0043 & SLAMF1, CD84 \\
\hline 736 & 0.0014 & 0.0043 & SCARB1, PDZK1 \\
\hline 895 & 0.0014 & 0.0043 & TRIM16, SETDB1 \\
\hline 851 & 0.0018 & 0.0043 & EDA, FURIN \\
\hline 1106 & 0.0018 & 0.0043 & RAD9A, HUS1 \\
\hline 900 & 0.0021 & 0.0044 & VDR, ESR1, NRIP1 \\
\hline 1041 & 0.0023 & 0.0044 & SCN4A, DGKZ \\
\hline 1077 & 0.0045 & 0.0078 & TREM2, KLRC3 \\
\hline 846 & 0.0052 & 0.0082 & IDH3B, ALDH1B1, TUBA1B \\
\hline 789 & 0.0083 & 0.0121 & $\mathrm{CFB}, \mathrm{C} 7$ \\
\hline 934 & 0.0344 & 0.0467 & PKP2, CDH15 \\
\hline
\end{tabular}

Table 2. Functional classification of different expression genes.

\begin{tabular}{lllll}
\hline GID & Bio-process & P value & Adjusted P & Genes \\
\hline GO:0030522 & Intracellular receptor-mediated signaling pathway & rawP $=0.0002$ & $\operatorname{adjP}=0.0456$ & ESR1, VDR, NRIP1 \\
GO:0051702 & Interaction with symbiont & rawP $=0.0004$ & $\operatorname{adjP}=0.0456$ & SCARB1, ALB \\
GO:0048385 & Regulation of retinoic acid receptor signaling pathway & $\operatorname{rawP}=0.0004$ & $\operatorname{adjP}=0.0456$ & TRIM16, ESR1 \\
GO:0015774 & Polysaccharide transport & $\operatorname{rawP}=0.0004$ & $\operatorname{adjP}=0.0456$ & SCARB1 \\
GO:0048384 & Retinoic acid receptor signaling pathway & rawP $=0.0004$ & $\operatorname{adjP}=0.0456$ & TRIM16, ESR1, ALDH1A2 \\
GO:0048386 & Positive regulation of retinoic acid receptor signaling pathway & rawP $=0.0004$ & $\operatorname{adjP}=0.0456$ & TRIM16, ESR1 \\
GO:0015920 & Lipopolysaccharide transport & rawP $=0.0004$ & $\operatorname{adjP}=0.0456$ & SCARB1 \\
\hline
\end{tabular}



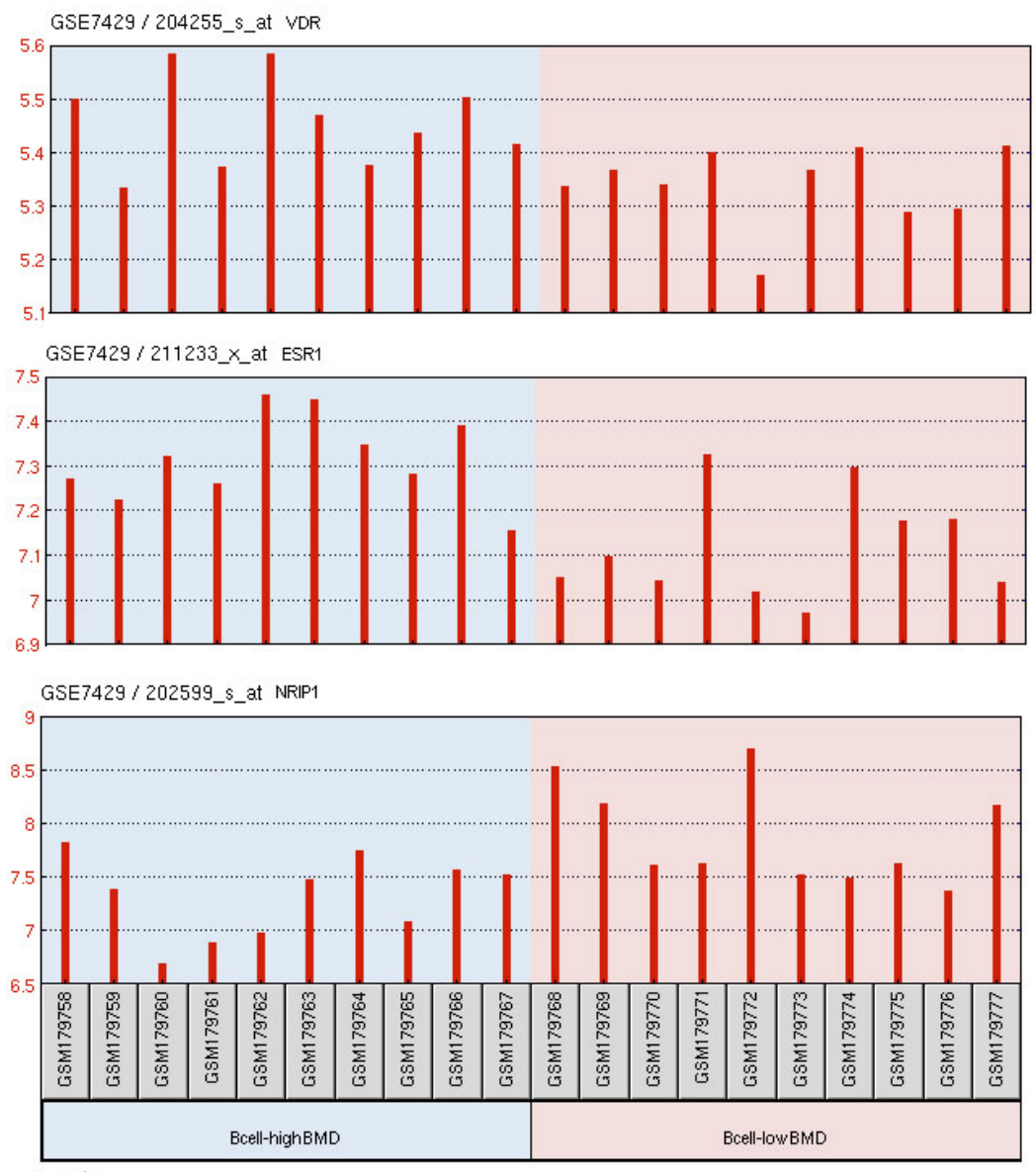

Figure 3. Original expressional data of 3 genes in different groups (top to bottom: VDR, ESR1, and NRIP1). $\mathrm{X}$-coordinate indicates different groups (blue box: high bone mineral density; pink box: low bone mineral density) and sample names. Y-coordinate indicates gene expression level.

\section{DISCUSSION}

Osteoporosis is a common condition of elderly men and women, which manifests clinically by minimal trauma fractures, particularly vertebral and hip fracture (Duncan and Brown, 2008). From family histories, twin studies, and molecular genetics, it is quite evident now that some of the predisposition for osteoporosis can be inherited. A large number of candidate genes have been identified in one study or another as being associated with osteoporosis. Most genetic studies in osteoporosis to date have focused on the genes of transforming growth factor and growth factors known to be involved in bone turnover (Bertoldo et al., 2000; Ben Amor et al., 2012), bone component gene (Weichetova et al., 2000), and hormones and their receptors that are related to bone metabolism (Horner, 2009; Horwitz et al., 2010). Among these candidate genes, VDR and ESR1 have received more attention (Kung et al., 
1998), which was also the case in our study when comparing the mRNA expression of blood B cells from different bone mineral density samples.

Vitamin D has been demonstrated to be responsible for intestinal calcium absorption and bone metabolism. Vitamin D deficiency can precipitate or exacerbate osteopenia and osteoporosis, cause osteomalacia and muscle weakness, and increase the risk of fracture (Holick, 2007). Emerging evidence suggests the potential ability of vitamin D exerts important roles in osteoporosis through interaction with its nuclear receptor (VDR ) (Holick and Chen, 2008). The VDR gene is also significantly associated with bone mineral density [22]. For example, the VDR BBAAtt haplotype has been observed in $23.94 \%$ of osteoporosis patients and $5.26 \%$ of controls. This haplotype is predicted to be a risk factor for osteoporosis, since an odds ratio (OR) of 5.66 has been found (Zambrano-Morales et al., 2008). VDR Apa I gene polymorphism is responsible for an important part of the heritable component of lumbar spine BMD in postmenopausal women, possibly related to impaired calcium absorption from the bowel (Dundar et al., 2009). VDR genotype-dependent increased fracture risk is especially pronounced in interaction with the COLIA1 genotype (Uitterlinden et al., 2001).

Endogenous estrogen is quite well known to exert an osteoprotective effect and indeed post-menopausal women with estrogen deficiency suffer osteoporotic defects in bone (Kato, 2009; Khosla, 2010). Numerous population genetic studies have provided evidence of an association between ESR1 polymorphisms and osteoporotic risk (Liu et al., 2006). A mutant ESR1 gene has been found to result in reduced BMD in humans (Smith et al., 1994) and mice (Delhon et al., 2009). In addition, the ESR1 gene is also positively correlated with the expression of the IGF1 (insulin-like growth factor 1) gene (Mendez et al., 2006) and Runx2 (Khalid et al., 2008), another important osteogenic growth factor that enhances the function of osteoblasts and prevents osteoblastic apoptosis. In the estrogen receptor signaling pathway, ESR1 positively regulates the activation of MAPK3 (Levin, 2003), which increases the activation of the nucleus ESR1-estrogen dimer (Likhite et al., 2006). Downregulation of ESR 1 and MAPK 3 in B cells controls the secretion of factors, leading to increased osteoclastogenesis or decreased osteoblastogenesis (Xiao et al., 2008).

NRIP1 (also known as RIP140) is a nuclear protein that specifically interacts with the hormone-dependent activation domain AF2 of nuclear receptors. This protein modulates transcriptional activity of the estrogen receptor (Augereau et al., 2006). Digenic genotypes involving ESR2-NRIP1 genes are strongly associated with osteoporosis (Morón et al., 2006).

Although it is controversial whether genetic determinants contribute to osteoporotic fracture, there is no doubt that multiple genes play an important role in the cause of osteoporosis (Albagha and Ralston, 2006). In this study, 2 lower expression level genes (VDR and ESR1) and 1 higher expression level gene (NRIP1) were identified in B cells of the osteoporosis sample. All these genes play important roles in blocking the decrease in BMD by interacting with their ligands. Our results reveal a new molecular technology for screening those at high-risk for osteoporosis.

\section{Conflicts of interest}

The authors have no conflict of interest to declare.

\section{REFERENCES}

Albagha OM and Ralston SH (2006). Genetics and osteoporosis. Rheum. Dis. Clin. North Am. 32: 659-680. 
Augereau P, Badia E, Fuentes M, Rabenoelina F, et al. (2006). Transcriptional regulation of the human NRIP1/RIP140 gene by estrogen is modulated by dioxin signalling. Mol. Pharmacol. 69: 1338-1346.

Ben Amor IM, Edouard T, Glorieux FH, Chabot G, et al. (2012). Low bone mass and high material bone density in two patients with Loeys-Dietz syndrome caused by transforming growth factor beta receptor 2 mutations. J. Bone Miner. Res. 27: 713-718.

Benjamini Y and Hochberg Y (1995). Controlling the false discovery rate: a practical and powerful approach to multiple testing. J. Royal Stat. Soc. Series B 57: 289-300.

Bertoldo F, D'Agruma L, Furlan F, Colapietro F, et al. (2000). Transforming growth factor- $\beta 1$ gene polymorphism, bone turnover, and bone mass in Italian postmenopausal women. J. Bone Miner. Res. 15: 634-639.

Cheung CL, Chan BY, Chan V, Ikegawa S, et al. (2009). Pre-B-cell leukemia homeobox 1 (PBX1) shows functional and possible genetic association with bone mineral density variation. Hum. Mol. Genet. 18: 679-687.

Delhon I, Gutzwiller S, Morvan F, Rangwala S, et al. (2009). Absence of estrogen receptor-related- $\alpha$ increases osteoblastic differentiation and cancellous bone mineral density. Endocrinology 150: 4463-4472.

Duncan EL and Brown MA (2008). Genetic studies in osteoporosis-the end of the beginning. Arthritis Res. Ther. 10: 214.

Duncan EL, Danoy P, Kemp JP, Leo PJ, et al. (2011). Genome-wide association study using extreme truncate selection identifies novel genes affecting bone mineral density and fracture risk. PLoS Genet. 7: e1001372.

Dundar U, Solak M, Kavuncu V, Ozdemir M, et al. (2009). Evidence of association of vitamin D receptor Apa I gene polymorphism with bone mineral density in postmenopausal women with osteoporosis. Clin. Rheumatol. 28: 11871191.

Fujita A, Sato JR, Rodrigues LO, Ferreira CE, et al. (2006). Evaluating different methods of microarray data normalization. BMC Bioinformatics 7: 469.

Holick MF (2007). Vitamin D deficiency. N. Engl. J. Med. 357: 266-281.

Holick MF and Chen TC (2008). Vitamin D deficiency: a worldwide problem with health consequences. Am. J. Clin. Nutr. 87: 1080S-1086S.

Horner KC (2009). The effect of sex hormones on bone metabolism of the otic capsule - an overview. Hear. Res. 252: 56-60.

Horwitz MJ, Tedesco MB, Garcia-Ocana A, Sereika SM, et al. (2010). Parathyroid hormone-related protein for the treatment of postmenopausal osteoporosis: defining the maximal tolerable dose. J. Clin. Endocrinol. Metab. 95: 1279-1287.

Hwang JY, Kim SY, Lee SH, Kim GS, et al. (2010). Association of TWIST1 gene polymorphisms with bone mineral density in postmenopausal women. Osteoporos. Int. 21: 757-764.

Kato S (2009). Hormones and osteoporosis update. Estrogen and bone remodeling. Clin. Calcium 19: 951-956.

Khalid O, Baniwal SK, Purcell DJ, Leclerc N, et al. (2008). Modulation of Runx2 activity by estrogen receptor- $\alpha$ : implications for osteoporosis and breast cancer. Endocrinology 149: 5984-5995.

Khosla S (2010). Update on estrogens and the skeleton. J. Clin. Endocrinol. Metab. 95: 3569-3577.

Kiel DP, Demissie S, Dupuis J, Lunetta KL, et al. (2007). Genome-wide association with bone mass and geometry in the Framingham Heart Study. BMC Med. Genet. 8 (Suppl 1): S14.

Kung AW, Yeung SS and Lau KS (1998). Vitamin D receptor gene polymorphisms and peak bone mass in southern Chinese women. Bone 22: 389-393.

Kung AW, Xiao SM, Cherny S, Li GH, et al. (2010). Association of JAG1 with bone mineral density and osteoporotic fractures: a genome-wide association study and follow-up replication studies. Am. J. Hum. Genet. 86: 229-239.

Levin ER (2003). Bidirectional signaling between the estrogen receptor and the epidermal growth factor receptor. Mol. Endocrinol. 17: 309-317.

Likhite VS, Stossi F, Kim K, Katzenellenbogen BS, et al. (2006). Kinase-specific phosphorylation of the estrogen receptor changes receptor interactions with ligand, deoxyribonucleic acid, and coregulators associated with alterations in estrogen and tamoxifen activity. Mol. Endocrinol. 20: 3120-3132.

Liu YJ, Shen H, Xiao P, Xiong DH, et al. (2006). Molecular genetic studies of gene identification for osteoporosis: a 2004 update. J. Bone Miner. Res. 21: 1511-1535.

Mencej-Bedrač S, Prezelj J, Kocjan T, Teskac K, et al. (2009). The combinations of polymorphisms in vitamin D receptor, osteoprotegerin and tumour necrosis factor superfamily member 11 genes are associated with bone mineral density. J. Mol. Endocrinol. 42: 239-247.

Mendez P, Wandosell F and Garcia-Segura LM (2006). Cross-talk between estrogen receptors and insulin-like growth factor-I receptor in the brain: cellular and molecular mechanisms. Front Neuroendocrinol. 27: 391-403.

Morón FJ, Mendoza N, Vazquez F, Molero E, et al. (2006). Multilocus analysis of estrogen-related genes in Spanish postmenopausal women suggests an interactive role of ESR1, ESR2 and NRIP1 genes in the pathogenesis of osteoporosis. Bone 39: 213-221. 
Nogales-Cadenas R, Carmona-Saez P, Vazquez M, Vicente C, et al. (2009). GeneCodis: interpreting gene lists through enrichment analysis and integration of diverse biological information. Nucleic Acids Res. 37: W317-W322.

Peacock M, Turner CH, Econs MJ and Foroud T (2002). Genetics of osteoporosis. Endocr. Rev. 23: 303-326.

Singh M, Singh P, Singh S, Juneja PK, et al. (2010). A susceptible haplotype within APOE gene influences BMD and intensifies the osteoporosis risk in postmenopausal women of Northwest India. Maturitas 67: 239-244.

Smith EP, Boyd J, Frank GR, Takahashi H, et al. (1994). Estrogen resistance caused by a mutation in the estrogen-receptor gene in a man. N. Engl. J. Med. 331: 1056-1061.

Tabas-Madrid D, Nogales-Cadenas R and Pascual-Montano A (2012). GeneCodis3: a non-redundant and modular enrichment analysis tool for functional genomics. Nucleic Acids Res. 40: W478-W483.

Troyanskaya O, Cantor M, Sherlock G, Brown P, et al. (2001). Missing value estimation methods for DNA microarrays. Bioinformatics 17: 520-525.

Uitterlinden AG, Weel AE, Burger H, Fang Y, et al. (2001). Interaction between the vitamin D receptor gene and collagen type I $\alpha 1$ gene in susceptibility for fracture. J. Bone Miner. Res. 16: 379-385.

Vidal C, Formosa R and Xuereb-Anastasi A (2011). Functional polymorphisms within the TNFRSF11B (osteoprotegerin) gene increase the risk for low bone mineral density. J. Mol. Endocrinol. 47: 327-333.

Weichetova M, Stepan JJ, Michalska D, Haas T, et al. (2000). COLIA1 polymorphism contributes to bone mineral density to assess prevalent wrist fractures. Bone 26: 287-290.

Wootton JC and Federhen S (1993). Statistics of local complexity in amino acid sequences and sequence databases. Comput. Chem. 17: 149-163.

Xiao P, Chen Y, Jiang H, Liu YZ, et al. (2008). In vivo genome-wide expression study on human circulating B cells suggests a novel ESR1 and MAPK3 network for postmenopausal osteoporosis. J. Bone Miner. Res. 23: 644-654.

Xiao SM, Gao Y, Cheung CL, Bow CH, et al. (2012). Association of CDX1 binding site of periostin gene with bone mineral density and vertebral fracture risk. Osteoporos. Int. 23: 1877-1887.

Yang TL, Guo Y, Liu YJ, Shen H, et al. (2012). Genetic variants in the SOX6 gene are associated with bone mineral density in both Caucasian and Chinese populations. Osteoporos. Int. 23: 781-787.

Zambrano-Morales M, Borjas L, Fernandez E, Zabala W, et al. (2008). Association of the vitamin D receptor gene BBAAtt haplotype with osteoporosis in post-menopausic women. Invest. Clin. 49: 29-38.

Zhang B, Kirov S and Snoddy J (2005). WebGestalt: an integrated system for exploring gene sets in various biological contexts. Nucleic Acids Res. 33: W741-W748. 